\title{
Safety metric profiling in surgery for temporal glioblastoma: lobectomy as a supra-total resection regime preserves perioperative standard quality rates
}

\author{
Matthias Schneider ${ }^{1}$ (D - Inja Ilic ${ }^{1}$. Anna-Laura Potthoff ${ }^{1} \cdot$ Motaz Hamed $^{1} \cdot$ Niklas Schäfer $^{2} \cdot$ Markus Velten $^{3}$. \\ Erdem Güresir $^{1}$ - Ulrich Herrlinger ${ }^{2} \cdot$ Valeri Borger $^{1} \cdot$ Hartmut Vatter $^{1} \cdot$ Patrick Schuss $^{1}$
}

Received: 30 July 2020 / Accepted: 21 September 2020 / Published online: 29 September 2020

(C) The Author(s) 2020

\begin{abstract}
Introduction Supra-total resection in terms of anterior temporal lobectomy (ATL) has gained growing attention with regard to superior long-term disease control for temporal-located glioblastoma. However, aggressive onco-surgical approaches-geared beyond conventional gross total resections (GTR) — may be associated with peri- and postoperative unfavorable events which significantly worsen initial favorable postoperative outcome. In the current study we analyzed our institutional database with regard to patient safety indicators (PSIs), hospital-acquired conditions (HACs) and specific cranial surgery-related complications (CSC) as high standard quality metric profiles in patients that had undergone surgery for temporal glioblastoma. Methods Between 2012 and 2018, 61 patients with temporal glioblastoma underwent GTR or temporal lobectomy at the authors' institution. Both groups of differing resection modalities were analyzed with regard to the incidence of PSIs, HACs and CSCs.

Results Overall, we found 6 PSI and 2 HAC events. Postoperative hemorrhage (3 out of 61 patients; 5\%) and catheter-associated urinary tract infection ( 2 out 61 patients; $3 \%$ ) were identified as the most frequent PSIs and HACs. PSIs were present in 1 out of 41 patients (5\%) for the temporal GTR and 2 out of 20 patients for the lobectomy group $(\mathrm{p}=1.0)$. Respective rates for PSIs were 5 of $41(12 \%)$ and 1 of $20(5 \%)(p=0.7)$. Further, CSCs did not yield significant differences between these two resection modalities $(\mathrm{p}=1.0)$.

Conclusion With regard to ATL and GTR as differing onco-surgical approaches these data suggest ATL in terms of an aggressive supra-total resection strategy to preserve perioperative standard safety metric profiles.
\end{abstract}

Keywords Anterior temporal lobectomy $\cdot$ Gross total resection $\cdot$ Postoperative complication profiling $\cdot$ Temporal glioblastoma surgery

Matthias Schneider and Inja Ilic contributed equally to this work.

Matthias Schneider

matthias.schneider@ukbonn.de

1 Department of Neurosurgery, University Hospital Bonn, Venusberg-Campus 1, 53127 Bonn, Germany

2 Division of Clinical Neurooncology, Department of Neurology, University Hospital Bonn, Bonn, Germany

3 Department of Anesthesiology, University Hospital Bonn, Bonn, Germany

\section{Introduction}

In regard of the fatal micro-invasive growth pattern of glioblastoma with histological evidence of malignant cells distant from magnetic resonance imaging (MRI)-detectable abnormalities, the perception of extended resection regimes far beyond the enhancing tumor bulk lesions increasingly is evolving within the field of surgical neuro-oncology [1-4]. Thus, extended supra-marginal glioblastoma resection should provide for superior long-term disease control and several studies have reported a survival benefit in these extended-resected patient cohorts, so far [5-7]. Recently, it has been demonstrated that in temporal glioblastoma disease resection of the entire anterior temporal lobe as a maximum variant of such supra-total conceptual approaches yielded 
significant prolonged median progression-free survival (mPFS) and median overall survival (mOS) rates therefore suggesting anterior temporal lobectomy (ATL) to constitute the surgical modality of choice for temporal-located glioblastoma [8]. However, given a potential increased impairment of eloquent areas and critical vasculature at risk, supra-marginal resection regimes might be accompanied by elevated levels of postoperative unfavorable events which in turn significantly worsen patients' postoperative quality of life [9]. Against this backdrop the present study was aimed at comparatively analyzing temporal GTR and ATL as differing onco-surgical resection modalities with regard to the onset of early postoperative unfavorable events in the course of temporal glioblastoma surgery.

\section{Methods}

\section{Patients and study design}

All patients aged $\geq 18$ years that underwent surgical resection of newly diagnosed temporal glioblastoma at the authors' department were entered in a computerized database (SPSS, version 25, IBMCorp., Armonk, NY). Surgical resection strategies were divided into the group of temporal gross total resection of the contrast-enhancing tumor manifestation as well as the group of anterior temporal lobectomy. In case of temporal lobectomy as a supra-total onco-surgical resection strategy, the posterior margin of the resection was approximately 5-6 cm from the temporal tip on the nondominant hemisphere, and $4-5 \mathrm{~cm}$ on the dominant side as described as a highly-standardized protocol for temporal lobe epilepsy surgery [10]. With regard to the retrospective study design, determination of temporal GTR or ATL as differing neurosurgical resection procedures was primarly based upon the prevalence of the particular neurosurgeon. Thus, ATL was performed only by neurosurgeons that were familiar to epilepsy surgical skills. Every neurosurgeon involved in the surgical treatment of the patients included in the present series fulfilled the requirements for NeuroOncology Centers certificated by the German Cancer Society. Patients with temporal glioblastoma were only included if the temporal tumor manifestation was precisely within the range of $4-5 \mathrm{~cm}$ on the dominant hemisphere and 5-6 cm on the nondominant hemisphere from the temporal tip including those with additional temporomesial infiltration (hippocampus, amygdala, parahipocampal gyrus and/or entorhinal cortex) objectified by means of preoperative gadolinium enhanced MR-imaging with identical sequence programs on a 3.0-T scanner (Achieva TX, Philips Healthcase, Best, Netherlands). Extent of resection was determined based on gadolinium-enhanced MR-imaging within $72 \mathrm{~h}$ after surgery. Gross total resection was defined as a complete resection without any residual nodular enhancement. Only patients with GTR or anterior temporal lobectomy were included for further analysis. Histopathological grading was performed according to the $2016 \mathrm{WHO}$ criteria [11]. MGMT promotor methylation status was analyzed using pyrosequencing and combined bisulfite restriction analysis [12]. This study was in compliance with the Helsinki Declaration and approved by the local institutional ethics committee. In regard to the retrospective study design, ethical approval was granted without the need for obtaining patient consent.

Pertinent clinical information including coexistence of arterial hypertension, coronary artery disease, atrial fibrillation, diabetes mellitus, antiplatelet and anticoagulant medication intake prior to surgery as well as history of pulmonary embolism (PE)/deep vein thrombosis (DVT) were assessed and further analyzed. Karnofsky performance scale (KPS) was used to grade patients' functional status at admission. Further stratification was made by means of the American Society of Anesthesiologists (ASA) classification into a group with preoperative ASA 1 or 2 as well as a group with ASA 3 or 4.

\section{Screening for postoperative complications}

In order to reach for highly standardized quality metric rating and profiling, analysis was done by means of a public-available list of events termed patient safety indicators (PSIs) and hospital-acquired conditions (HACs) introduced by the Agency of Healthcare Research and Quality and the Center for Medicare and Medicaid Services (Fig. 1) [13, 14]. Thereby, PSIs entailed pressure ulcer, iatrogenic pneumothorax, central venous catheter-related blood stream infection, transfusion reaction, retained surgical item, peri- and postoperative hemorrhage, acute postoperative respiratory failure, pulmonary embolism, deep venous thrombosis (DVT), postoperative sepsis, wound dehiscence, accidental puncture or laceration, postoperative hip fracture as well as postoperative physiologic and metabolic derangement. Within the group of HACs, screening was performed for foreign object retained after surgery, air embolism, blood incompatibility, pressure ulcer states II and IV, catheter-associated urinary tract infection, fracture, dislocation, intracranial injury, crushing injury, vascular catheter-associated infection and manifestation of poor glycemic control (diabetic ketoacidosis, nonketonic hyperosmolar coma, hyperglycemic coma). Postoperative periods were additionally screened for iatrogenic ischaemic infarction, cerebro spinal fluid (CSF) leakage, postoperative meningitis and ventriculitis as well as postoperative new or worsened neurological deficits including postoperative new speech and language deficits and subsequently classified as specific cranial surgery-related complications (CSCs). 

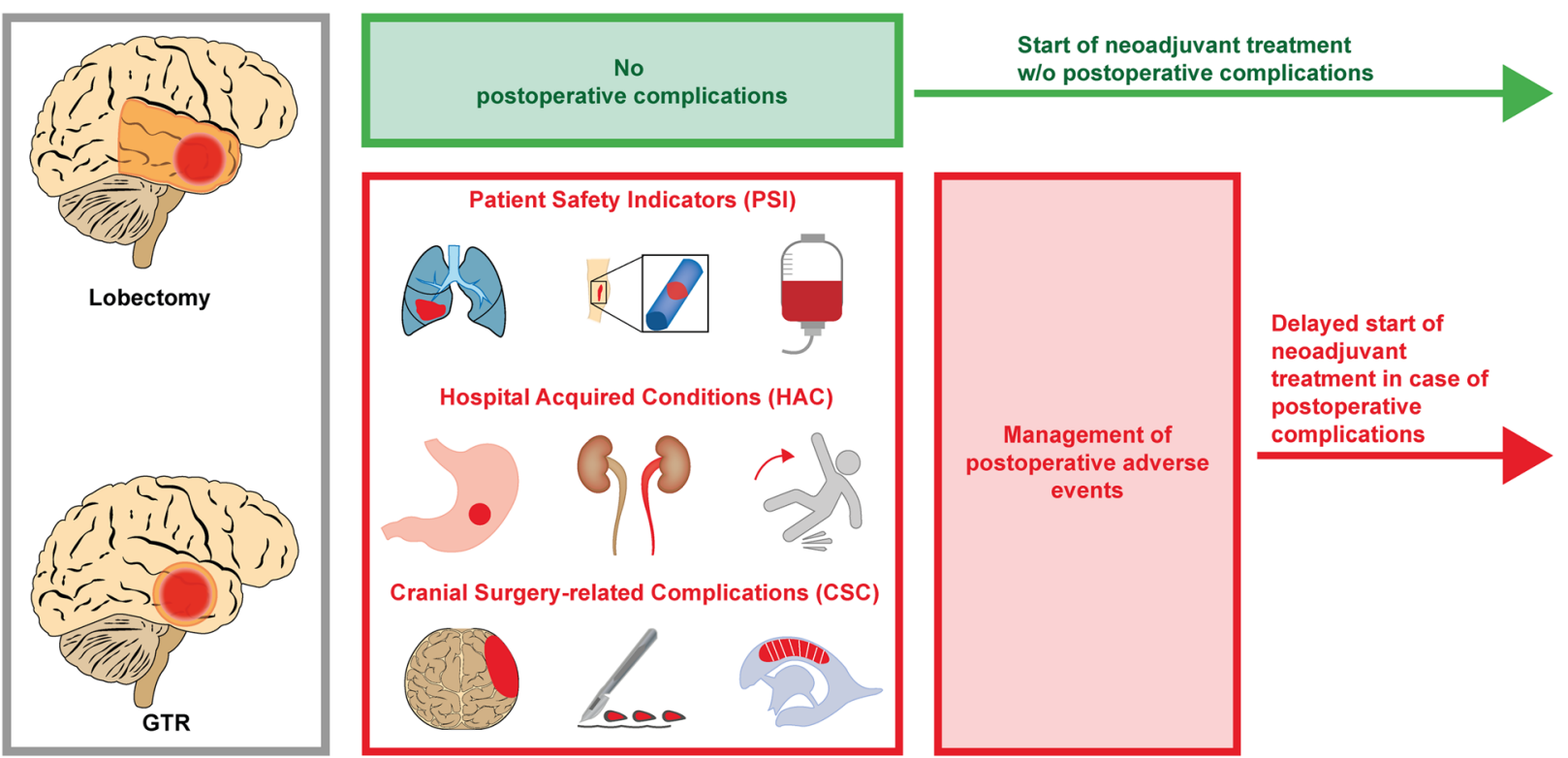

Fig. 1 Graphical synopsis of safety metric profiling in temporal glioblastoma surgery

Occurrence of early postoperative complications served as primary readout and was defined as any postoperative unfavorable event that appeared within 30 days following initial temporal glioblastoma resection.

\section{Statistics}

Data analyses were performed using the computer software package SPSS (version 25, IBM Corp., Armonk, NY). Categorical variables were analyzed in contingency tables using Fisher's exact test. For discrete and continuous variables mean and standard deviation (SD) was determined unless stated otherwise. Results with $\mathrm{p}<0.05$ were considered statistically significant.

\section{Results}

\section{Baseline characteristics}

Between 2012 and 2018, 61 patients were surgically treated for temporal-located glioblastoma at the authors' institution. Preoperative MR-imaging revealed tumor manifestation located precisely within the temporal lobe in 39 patients (64\%), whereas 22 patients (36\%) exhibited additional temporomesial malignant infiltration. Anterior temporal lobectomy and temporal GTR were performed in 20 (33\%) and 41 patients (67\%), respectively. Overall, early postoperative unfavorable events following resection of temporal
Table 1 Baseline characteristics

\begin{tabular}{ll}
\hline Sex & $\mathrm{n}$ \\
Male & $39(64)$ \\
Female & $22(36)$ \\
Mean age $( \pm$ SD) (in yrs) & $63 \pm 13$ \\
Tumor location & \\
Temporomesial infiltration (-) & $39(64)$ \\
Temporomesial infiltration (+) & $22(36)$ \\
Preoperative KPS & \\
$\geq 70$ & $47(77)$ \\
<70 & $14(23)$ \\
Surgical modality & \\
Temporal lobectomy & $20(33)$ \\
Temporal GTR & $41(67)$ \\
MGMT promotor methylation status & \\
Methylated & $22(43)$ \\
Unmethylated & $29(57)$ \\
IDH mutation status & \\
Wild type & $37(97)$ \\
Mutant & $1(3)$ \\
Postoperative complications & $10(16)$ \\
\hline
\end{tabular}

Values represent number of patients unless otherwise indicated (\%) $d s$ days, GTR gross total resection, KPS Karnofsky performance scale, $S D$ standard deviation, yrs years

glioblastoma were present in 10 out of 61 patients (16\%). For further details see Table 1. Patients with GTR exhibited a mOS rate of 12 months (95\% CI 5-14) compared 
to 23 months (95\% CI 16-33) for patients with ATL $(\mathrm{p}=0.0005)$.

\section{Early postoperative complications}

Analysis of early postoperative complications revealed subclassification into 6 PSIs, 2 HACs as well as 2 specific CSCs (Table 2). Postoperative hemorrhage and catheter-associated urinary tract infection were the most frequent PSIs and HACs accounting for $5 \%$ and $3 \%$ of patients within the overall cohort of temporal glioblastoma patients, respectively. Among the patients with postoperative hemorrhage, revision surgery was required for one subject. Postoperative sepsis, wound dehiscence and pulmonary embolism were identified as further PSIs in 1 out of 61 patients (2\%) each. The subgroup of specific CSCs was made up of postoperative CSF leakage and meningitis accounting for 1 patient (2\%), respectively. No profound new language or visual deficit was seen within the routine follow up examinations.

\section{Safety metric profiling of ATL and temporal GTR as differing onco-surgical resection modalities}

In order to sufficiently compare safety metric profiles of anterior temporal lobectomy and temporal GTR by means of postoperative complication rates, both strategies of temporal glioblastoma resection were first analyzed for potential patient- and tumor-related covariates. Comparative analysis of preoperative KPS, KPS at discharge and ASA-scoring as well as comorbidities such as arterial hypertension, coronary artery disease and diabetes mellitus among others revealed homogeneous distribution of risk factor profiles in both these groups of differing resection strategies (Table 3). Similarly, presence of temporomesial malignant infiltration,

Table 2 Overview of postoperative complications

\begin{tabular}{ll}
\hline No. of patients & 61 \\
No. of complications & $10(16)$ \\
PSIs & $6(10)$ \\
Postoperative hemorrhage & $3(5)$ \\
Postoperative sepsis & $1(2)$ \\
Wound dehiscence & $1(2)$ \\
Perioperative pulmonary embolism & $1(2)$ \\
HACs & $2(3)$ \\
Catheter-associated urinary tract infection & $2(3)$ \\
Specific CSCs & $2(3)$ \\
CSF leakage & $1(2)$ \\
Meningitis & $1(2)$ \\
\hline
\end{tabular}

Values represent number of patients (\%) unless otherwise indicated CSCs cranial surgery-related complications, CSF cerebro spinal fluid, HACs hospital-acquired conditions, No. number of patients, PSIs patient safety indicators mean operation time, mean hospital stay and postoperative new seizure onset within 3 months after surgery were evenly distributed. Volumetric analysis of preoperative tumor and postoperative resection cavity volumes could objectify intended supratotal extent of resection for anterior temporal lobectomies: while mean resection cavity volumes revealed $29 \mathrm{~cm}^{3}$ for temporal GTR versus $58 \mathrm{~cm}^{3}$ for temporal lobectomy ( $\mathrm{p}=0.0001)$, preoperative tumor volumes did not significantly differ for these two resection modalities $\left(31 \mathrm{~cm}^{3}\right.$ vs. $33 \mathrm{~cm}^{3}, \mathrm{p}=0.8$ ).

Having demonstrated equal distribution of potential interfering cofounders, ATL and temporal GTR subsequently were compared for the rate of postoperative early unfavorable events. PSIs were present in 5 out of 41 patients (12\%) for the temporal GTR and 1 out of 20 patients (5\%) for the lobectomy group $(p=0.7)$ (Table 3$)$. Respective rates for HACs were 1 out of $41(2 \%)$ and 1 out of $20(5 \%)(p=1.0)$. Analysis of specific cranial CSCs yielded 1 out of $41(2 \%)$ patients with postoperative CSF-leakage in the GTR group as well as 1 out of 20 patients (5\%) with postoperative meningitis in the lobectomy group $(\mathrm{p}=1.0)$. In summary, PSIs, HACs and specific CSCs did not significantly differ between these two groups of differing onco-surgical approaches.

\section{Discussion}

Given the growing evidence of a greater extent of malignant primary brain tumor resection to entail significantly prolonged time to tumor progression and superior overall survival rates, the concept of supra-total resection strategies far beyond tumoral enhancing MRI abnormalities is increasingly emerging in the field of neurosurgical oncology [6, 15-17]. In a recent study, we could demonstrate that-in temporal glioblastoma disease-ATL as a paradigm for such supra-marginal extended surgery regimes was accompanied by a significant survival benefit compared to conventional gadolinium- and 5-ALA-guided temporal GTRs [8]. However, the aim of aggressive extended surgical approaches has to surrender the risk of potential elevated levels of postoperative complications [9]. Patients who suffer from postoperative unfavorable events following malignant brain tumor resection have been shown to exhibit profound worsened long-term quality of life as well as to be at a more than 4-times higher risk for in-hospital mortality [9, 18]. Furthermore, postoperative complications have been reported to be accompanied by a delay of adjuvant chemotherapy therefore additionally reducing oncological outcomes (Fig. 1) [19, 20]. In the present study, we therefore analyzed ATL and temporal GTR as differing onco-surgical resection modalities for temporal-located glioblastoma in view of the incidence of postoperative early complications. In order to achieve a comprehensive assessment of overall postoperative complication 
Table 3 Safety metric profiling of ATL and temporal GTR

\begin{tabular}{|c|c|c|c|}
\hline & $\begin{array}{l}\text { Temporal lobectomy } \\
20(33)\end{array}$ & $\begin{array}{l}\text { Temporal GTR } 41 \\
\text { (67) }\end{array}$ & $\mathrm{p}$ value \\
\hline Mean age (yrs) & $61 \pm 12$ & $63 \pm 12$ & 0.2 \\
\hline Sex & & & 0.6 \\
\hline Female & $6(30)$ & $16(39)$ & \\
\hline Male & $14(70)$ & $25(61)$ & \\
\hline KPS preoperative & & & 1.0 \\
\hline$\geq 70$ & $16(80)$ & $31(76)$ & \\
\hline \multicolumn{4}{|l|}{ KPS at discharge } \\
\hline$\geq 70$ & $15(80)$ & 29 & 1.0 \\
\hline Tumor location & & & 0.3 \\
\hline Temporomesial infiltration (-) & $15(75)$ & $24(59)$ & \\
\hline Temporomesial infiltration $(+)$ & $5(25)$ & $17(41)$ & \\
\hline \multicolumn{4}{|l|}{ Volumetric analysis } \\
\hline Mean tumor volume & $33 \pm 21$ & $31 \pm 25$ & 0.8 \\
\hline Mean resection cavity volume & $58 \pm 15$ & $29 \pm 19$ & 0.0001 \\
\hline Mean operation time (min) & $270 \pm 97$ & $268 \pm 67$ & 0.9 \\
\hline Mean hospital stay (ds) & $14 \pm 7$ & $15 \pm 8$ & 0.6 \\
\hline Postoperative seizure onset ${ }^{\mathrm{a}}$ & $0(0)$ & $2(5)$ & 1.0 \\
\hline ASA score & & & 1.0 \\
\hline 1 or 2 & $15(75)$ & $31(76)$ & \\
\hline 3 or 4 & $5(25)$ & $10(24)$ & \\
\hline Anticoagulant medication prior to surgery & $4(20)$ & $4(10)$ & 0.4 \\
\hline \multicolumn{4}{|l|}{ Comorbidities } \\
\hline Arterial hypertension & $5(25)$ & $16(39)$ & 0.4 \\
\hline Coronary artery disease & $2(10)$ & $1(2)$ & 0.2 \\
\hline Atrial fibrillation & $1(5)$ & $1(2)$ & 1.0 \\
\hline Diabetes mellitus & $1(5)$ & $4(10)$ & 1.0 \\
\hline History of PE/DVT & $1(5)$ & $1(2)$ & 1.0 \\
\hline \multicolumn{4}{|l|}{ Postoperative complications } \\
\hline PSIs & $1(5)$ & $5(12)$ & 0.7 \\
\hline HACs & $1(5)$ & $1(2)$ & 1.0 \\
\hline Specific CSCs & $1(5)$ & $1(2)$ & 1.0 \\
\hline
\end{tabular}

Values are presented as the number of patients (\%) unless stated otherwise

ASA American Society of Anesthesiology, ATL anterior temporal lobectomy, CSCs cranial surgery-related complications, $d s$ days, DVT deep vein thrombosis, GTR gross total resection, HACs hospital-acquired conditions, KPS Karnofsky Performance Scale, min, minutes, No. number, PE pulmonary embolism, PSIs patient safety indicators, yrs years

${ }^{a}$ Within 3 months after surgery rates in terms of highly standardized quality metric profiling, screening for postoperative events was performed by means of PSIs and HACs as public-available quality rating classification tools $[13,14]$.

The overall morbidity rate was found to reach about $16 \%$. Thereby, the quantitative complication level in our series was in accordance to existent literature where respective values on overall complications in glioblastoma surgery are given between 11 and $32 \%$ [16, 21-23]. It is important to mention that a PSI- and HAC-based assessment of postoperative unfavorable events will also cover transient events like catheter-associated urinary tract infections and therefore will quantitatively surpass complication levels of several previous studies that focused exclusively on postoperative events which required further surgical treatment [21, 24, 25]. Further, postoperative secondary hemorrhage and urinary tract infection were identified as the most common unfavorable events within the presented patient cohort of selected temporal glioblastoma disease and observed levels of incidence met previously reported data [26-28].

Presented analysis of postoperative complications dependent on the surgical modality within temporal glioblastoma surgery did not reveal any indications to increased risk profiles in case of supra-total temporal glioblastoma 
resection. As against several concerns that have been raised on potential elevated risks in the context of far extended tumor resections, similar findings of unimpaired or even decreased levels of iatrogenic postoperative complications following supra-marginal glioblastoma resection have been reported for cohorts of topographically unselected glioblastoma locations [27]. Such preservation of quality metric profiles in the course of supra total glioblastoma resection might - among others - be reasoned in a reduced risk for the development of postoperative reactive peritumoral edema as well as a decreased risk of postoperative intratumoral bleeding from partially-resected vulnerable tumor remnants $[27,29]$. The current study is the first to provide data on postoperative complications of supra-total tumor resection in the setting of specifically temporal-located glioblastoma disease. In contrast to the inconsistent perception of the extent of supra-total tumor removal within the existing literature varying from resection beyond $\mathrm{T} 1$-enhanced tumor areas, but within the boundaries of FLAIR abnormalities, to resection beyond any visible MRI abnormality, ATL as a maximum variant of a supra total resection policy with excision of the entire anterior temporal lobe constitutes a highly-standardized and clearly-defined procedure $[8,30$, 31]. Therefore, subsequent rather multicenter studies will be capable not only to cope with the low frequency of temporal glioblastoma patients, but also to sufficiently evaluate the potential of ATL by means of intercenter comparison to supersede a mere complete tumor bulk removal and thus constitute the surgical modality of choice for temporallocated glioblastoma.

\section{Limitations}

The present study has several limitations. Acquisition of data was retrospective and therefore, determination of the surgical resection modality was based on the preference of the particular neurosurgeon and not randomized. Owed to the retrospective nature, a comprehensive assessment of visual field deficits by ophthalmologic examinations as well as specific language assessment tools were beyond the scope of the present manuscript. Further, though not statistically significant, given the trend of a higher rate of preoperative tumoral temporomesial infiltration within the ATL group, determination of the surgical resection modality might potentially not only be associated with neurosurgeon-specific preferences, but also with characteristics of tumor location. Given the highly-selective inclusion criteria of temporal-located glioblastoma patients, the study population was quite small and therefore might entail unconsidered further selection bias. Future multicenter studies might clarify such potential cofounding restraints. Additionally, the present data represent a single-center experience, only.

\section{Conclusions}

With regard to ATL and GTR that did not exhibit significant differences in the rates of peri- and postoperative unfavorable events in the course of temporal glioblastoma resection, our data suggest ATL in terms of a supra-total conceptual resection modality not to fail perioperative standard safety metric profiles. Given the previously reported survival benefit compared to a mere complete tumor bulk removal, ATL might constitute the surgical modality of choice for temporal-located glioblastoma.

Funding Open Access funding enabled and organized by Projekt DEAL. The authors received no financial support related to this study.

Data availability Restrictions apply to the availability of these data due to privacy restrictions.

\section{Compliance with ethical standards}

Conflict of interest The authors declare that they have no conflict of interest.

Ethical approval The present study was approved by the local ethics committee at the University of Bonn.

Informed consent Informed consent was not sought as a retrospective study design was used. All authors agreed to the publication of the manuscript.

Open Access This article is licensed under a Creative Commons Attribution 4.0 International License, which permits use, sharing, adaptation, distribution and reproduction in any medium or format, as long as you give appropriate credit to the original author(s) and the source, provide a link to the Creative Commons licence, and indicate if changes were made. The images or other third party material in this article are included in the article's Creative Commons licence, unless indicated otherwise in a credit line to the material. If material is not included in the article's Creative Commons licence and your intended use is not permitted by statutory regulation or exceeds the permitted use, you will need to obtain permission directly from the copyright holder. To view a copy of this licence, visit http://creativecommons.org/licenses/by/4.0/.

\section{References}

1. Kelly PJ, Daumas-Duport C, Kispert DB, Kall BA, Scheithauer BW, Illig JJ (1987) Imaging-based stereotaxic serial biopsies in untreated intracranial glial neoplasms. J Neurosurg 66:865-874. https://doi.org/10.3171/jns.1987.66.6.0865

2. Silbergeld DL, Chicoine MR (1997) Isolation and characterization of human malignant glioma cells from histologically normal brain. J Neurosurg 86:525-531. https://doi.org/10.3171/ jns.1997.86.3.0525

3. Brown TJ, Brennan MC, Li M, Church EW, Brandmeir NJ, Rakszawski KL, Patel AS, Rizk EB, Suki D, Sawaya R, Glantz M (2016) Association of the extent of resection with survival in glioblastoma: a systematic review and meta-analysis. 
JAMA Oncol 2:1460-1469. https://doi.org/10.1001/jamao ncol.2016.1373

4. Yan JL, van der Hoorn A, Larkin TJ, Boonzaier NR, Matys T, Price SJ (2017) Extent of resection of peritumoral diffusion tensor imaging-detected abnormality as a predictor of survival in adult glioblastoma patients. J Neurosurg 126:234-241. https:// doi.org/10.3171/2016.1.JNS152153

5. D'Amico RS, Englander ZK, Canoll P, Bruce JN (2017) Extent of resection in glioma - a review of the cutting edge. World Neurosurg 103:538-549. https://doi.org/10.1016/j.wneu.2017.04.041

6. Lacroix M, Abi-Said D, Fourney DR, Gokaslan ZL, Shi W, DeMonte F, Lang FF, McCutcheon IE, Hassenbusch SJ, Holland E, Hess K, Michael C, Miller D, Sawaya R (2001) A multivariate analysis of 416 patients with glioblastoma multiforme: prognosis, extent of resection, and survival. J Neurosurg 95:190-198. https ://doi.org/10.3171/jns.2001.95.2.0190

7. Sanai N, Polley MY, McDermott MW, Parsa AT, Berger MS (2011) An extent of resection threshold for newly diagnosed glioblastomas. J Neurosurg 115:3-8. https://doi. org/10.3171/2011.2.JNS10998

8. Schneider M, Potthoff AL, Keil VC, Güresir Á, Weller J, Borger V, Hamed M, Waha A, Vatter H, Güresir E, Herrlinger U, Schuss P (2019) Surgery for temporal glioblastoma: lobectomy outranks oncosurgical-based gross-total resection. J Neurooncol 145:143150. https://doi.org/10.1007/s11060-019-03281-1

9. Rahman M, Abbatematteo J, De Leo EK, Kubilis PS, Vaziri S, Bova F, Sayour E, Mitchell D, Quinones-Hinojosa A (2017) The effects of new or worsened postoperative neurological deficits on survival of patients with glioblastoma. J Neurosurg 127:123-131. https://doi.org/10.3171/2016.7.JNS16396

10. Wiebe S, Blume WT, Girvin JP, Eliasziw M, Effectiveness, Efficiency of Surgery for Temporal Lobe Epilepsy Study G (2001) A randomized, controlled trial of surgery for temporal-lobe epilepsy. N Engl J Med 345:311-318. https://doi.org/10.1056/NEJM200108 023450501

11. Louis DN, Perry A, Reifenberger G, von Deimling A, FigarellaBranger D, Cavenee WK, Ohgaki H, Wiestler OD, Kleihues P, Ellison DW (2016) The 2016 World Health Organization classification of tumors of the central nervous system: a summary. Acta Neuropathol 131:803-820. https://doi.org/10.1007/s0040 1-016-1545-1

12. Mikeska T, Bock C, El-Maarri O, Hubner A, Ehrentraut D, Schramm J, Felsberg J, Kahl P, Buttner R, Pietsch T, Waha A (2007) Optimization of quantitative MGMT promoter methylation analysis using pyrosequencing and combined bisulfite restriction analysis. J Mol Diagn 9:368-381. https://doi.org/10.2353/jmold x.2007.060167

13. Agency for Healthcare Research and Quality (2015) Patient Safety Indicators. https://www.qualityindicators.ahrq.gov/Downloads/ Modules/PSI/V50/PSI_Brochure.pdf. Accessed 24 May 2020

14. Centers for Medicare \& Medicaid Services (2020) HospitalAcquired Conditions. https://www.cms.gov/Medicare/Medic are-Fee-for-Service-Payment/HospitalAcqCond/Hospital-Acqui red_Conditions. Accessed 24 May 2020

15. Chaichana KL, Cabrera-Aldana EE, Jusue-Torres I, Wijesekera O, Olivi A, Rahman M, Quinones-Hinojosa A (2014) When gross total resection of a glioblastoma is possible, how much resection should be achieved? World Neurosurg 82:e257-265. https://doi. org/10.1016/j.wneu.2014.01.019

16. Almeida JP, Chaichana KL, Rincon-Torroella J, Quinones-Hinojosa A (2015) The value of extent of resection of glioblastomas: clinical evidence and current approach. Curr Neurol Neurosci Rep 15:517. https://doi.org/10.1007/s11910-014-0517-x

17. Grabowski MM, Recinos PF, Nowacki AS, Schroeder JL, Angelov L, Barnett GH, Vogelbaum MA (2014) Residual tumor volume versus extent of resection: predictors of survival after surgery for glioblastoma. J Neurosurg 121:1115-1123. https:// doi.org/10.3171/2014.7.JNS132449

18. De la Garza-Ramos R, Kerezoudis P, Tamargo RJ, Brem H, Huang J, Bydon M (2016) Surgical complications following malignant brain tumor surgery: an analysis of 2002-2011 data. Clin Neurol Neurosurg 140:6-10. https://doi.org/10.1016/j.cline uro.2015.11.005

19. Kim IY, Kim BR, Kim YW (2015) Factors affecting use and delay $(>/=8$ weeks $)$ of adjuvant chemotherapy after colorectal cancer surgery and the impact of chemotherapy-use and delay on oncologic outcomes. PLoS ONE 10:e0138720. https://doi.org/10.1371/ journal.pone. 0138720

20. Merkow RP, Bilimoria KY, Tomlinson JS, Paruch JL, Fleming JB, Talamonti MS, Ko CY, Bentrem DJ (2014) Postoperative complications reduce adjuvant chemotherapy use in resectable pancreatic cancer. Ann Surg 260:372-377. https://doi.org/10.1097/ SLA.0000000000000378

21. Laurent D, Freedman R, Cope L, Sacks P, Abbatematteo J, Kubilis P, Bova F, Rahman M (2020) Impact of extent of resection on incidence of postoperative complications in patients with glioblastoma. Neurosurgery 86:625-630. https://doi.org/10.1093/neuros/ nyz313

22. Sawaya R, Hammoud M, Schoppa D, Hess KR, Wu SZ, Shi WM, Wildrick DM (1998) Neurosurgical outcomes in a modern series of 400 craniotomies for treatment of parenchymal tumors. Neurosurgery 42:1044-1055. https://doi.org/10.1097/00006123-19980 5000-00054(discussion 1055-1046)

23. Hoffmann J, Westendorff C, Schneider M, Reinert S (2005) Accuracy assessment of image-guided implant surgery: an experimental study. Int J Oral Maxillofac Implants 20:382-386

24. Moghavem N, McDonald K, Ratliff JK, Hernandez-Boussard T (2016) Performance measures in neurosurgical patient care: differing applications of patient safety indicators. Med Care 54:359364. https://doi.org/10.1097/MLR.0000000000000490

25. Schneider M, Borger V, Grigutsch D, Guresir A, Potthoff AL, Velten M, Vatter H, Guresir E, Schuss P (2020) Elevated body mass index facilitates early postoperative complications after surgery for intracranial meningioma. Neurosurg Rev. https://doi. org/10.1007/s10143-020-01281-8

26. Asthagiri AR, Pouratian N, Sherman J, Ahmed G, Shaffrey ME (2007) Advances in brain tumor surgery. Neurol Clin 25:9751003. https://doi.org/10.1016/j.ncl.2007.07.006

27. Jackson C, Westphal M, Quinones-Hinojosa A (2016) Complications of glioma surgery. Handb Clin Neurol 134:201-218. https ://doi.org/10.1016/B978-0-12-802997-8.00012-8

28. Aghi MK, Batchelor TT, Louis DN, Barker FG 2nd, Curry WT Jr (2009) Decreased rate of infection in glioblastoma patients with allelic loss of chromosome 10q. J Neurooncol 93:115-120. https ://doi.org/10.1007/s11060-009-9826-3

29. McGirt MJ, Mukherjee D, Chaichana KL, Than KD, Weingart JD, Quinones-Hinojosa A (2009) Association of surgically acquired motor and language deficits on overall survival after resection of glioblastoma multiforme. Neurosurgery 65:463-469. https://doi. org/10.1227/01.NEU.0000349763.42238.E9(discussion 469-470)

30. Li YM, Suki D, Hess K, Sawaya R (2016) The influence of maximum safe resection of glioblastoma on survival in 1229 patients: can we do better than gross-total resection? J Neurosurg 124:977988. https://doi.org/10.3171/2015.5.JNS142087

31. de Leeuw CN, Vogelbaum MA (2019) Supratotal resection in glioma: a systematic review. Neuro Oncol 21:179-188. https:// doi.org/10.1093/neuonc/noy166

Publisher's Note Springer Nature remains neutral with regard to jurisdictional claims in published maps and institutional affiliations. 